\title{
Different Patterns of Endocytosis in Cochlear Inner and Outer Hair Cells of Mice
}

\author{
Sijun $\mathrm{LI}^{1 *}$, Shukui YU ${ }^{2} *$, Tonghui DING ${ }^{3}$, Aihui YAN ${ }^{3}$, Yue $\mathrm{QI}^{2}$, Shusheng GONG ${ }^{2}$, Siquan \\ TANG $^{1}$, Ke LIU $^{2}$
}

*These authors contributed equally to this work.

${ }^{1}$ Department of Otolaryngology-Head and Neck Surgery, Affiliated Hospital of North Sichuan Medical College, Nanchong, Sichuan, China, ${ }^{2}$ Department of Otolaryngology-Head and Neck Surgery, Beijing Friendship Hospital, Capital Medical University, Beijing, China, ${ }^{3}$ Department of Otolaryngology-Head and Neck Surgery, The First Affiliated Hospital, China Medical University, Shenyang, China*

Received August 26, 2018

Accepted February 26, 2019

Epub Ahead of Print June 6, 2019

\begin{abstract}
Summary
Precise and efficient endocytosis is critical for sustained neurotransmission during continuous neuronal activity. Endocytosis is a prerequisite for maintaining the auditory function. However, the differences between the patterns of endocytosis in cochlear inner hair cells (IHCs) and outer hair cells (OHCs) remain unclear. Both IHCs and OHCs were obtained from adult C57 mice. Patterns of endocytosis in cells were estimated by analyzing the uptake of FM1-43, a fluorescent. The observations were made using live confocal imaging, fluorescence intensities were calculated statistically. Results revealed the details about following phenomenon, i) sites of entry: the FM1-43 dye was found to enter IHC at the apical area initially, the additional sites of entry were then found at basolateral membrane of the cells, The entry of the dye into OHCs initially appeared to be occurring around whole apical membranes area, which then diffused towards the other membrane surface of the cells, ii) capacity of endocytosis: fluorescence intensity in IHCs showed significantly higher than that of OHCs $(P<0.01)$. We have found different patterns of endocytosis between IHCs and OHCs, this indicated functional distinctions between them. Moreover, FM1-43 dye can be potentially used as an indicator of the functional loss or repair of cochlear hair cells.
\end{abstract}

\section{Key words}

Endocytosis - Cochlea - Inner and outer hair cells • Mice • FM1-43 dye

\section{Corresponding authors}

Tang SQ, Department of Otolaryngology-Head and Neck Surgery, Affiliated Hospital of North Sichuan Medical College, 63 Wenhua, Road, Nanchong, Sichuan, China. Email: tangsiqu@126.com; Liu K, Department of Otolaryngology-Head and Neck Surgery, Beijing Friendship Hospital, Capital Medical University, 95 Yong'an Road, Beijing, 100050, China. Email: liuke@ccmu.edu.cn

\section{Introduction}

Dynamic endocytosis is crucial in maintaining physiological function of living cells, different capacities of endocytosis were proposed to largely indicate distinct function of cells (Cousin, 2017, Xie et al. 2017). During endocytosis or exocytosis, cells require an efficient membrane recycling to ensure the integrity of the auditory pathway. It is known that cochlear hair cells, which amplify and encode the sound signals, require fast and sustained rates of neurotransmitters release, and need higher rate of endocytosis (Meyer et al. 2001, Neef et al. 2014). Cochlear hair cells are composed of two types of sensory cells, namely, outer hair cells (OHCs) and inner hair cells (IHCs). OHCs serve as amplifier for the sound signals. IHCs are polarized neuroepithelial cells that have massive synaptic release sites around their basolateral membrane (Merchan-Perez and Liberman, 1996).

In past decades, endocytosis of IHCs and OHCs 
were investigated in depth using FM1-43 dye. FM1-43 dye was reported to reflect membrane recycling, and can serve as an indicator of endocytosis in cochlear hair cells in mammalian systems (Griesinger et al. 2004, Kamin et al. 2014). However, the differences in the patterns of endocytosis between IHCs and $\mathrm{OHCs}$ remain unexplored. Based on different structures and roles of OHCs and IHCs, we therefore assume that there are going to be different patterns of endocytosis in OHCs and IHCs in mice. Possible distinctions of endocytosis between OHCs and IHCs may suggest a functional contrast between OHCs and IHCs. In this study, we have explored the patterns of endocytosis in both $\mathrm{OHCs}$ and IHCs by analyzing the uptake of styryl dye FM1-43 using confocal microscope.

\section{Methods}

\section{Animal preparations}

5 adult (6 weeks old) C57 mice with normal hearing were employed in this experiments (data not shown), the animals were obtained from the Chinese Academy of Medical Sciences Animal Center (Beijing, China). The average body weight was $22 \pm 2 \mathrm{~g}$, absence of outer or middle ear pathologies was confirmed. This study was carried out in strict accordance with the recommendations in the Guide for the Care and Use of Laboratory Animals of the National Institutes of Health. All surgery was performed under $10 \%$ chloral hydrate anesthesia, and all possible efforts were made to minimize suffering. In this study, mice were handled according to the specifications of affiliated Hospital of North Sichuan Medical College and Beijing Friendship Hospital, Capital Medical University. The procedures were approved by affiliated Hospital of North Sichuan Medical College Board for Animal Welfare and the Animal Welfare Office of Beijing Friendship Hospital, Capital Medical University.

\section{Auditory evoked brainstem response (ABR) detections}

Mice were tested double blindly for ABR thresholds with equipment from Intelligent Hearing Systems (Miami, FL). TDT system III (Tucker-Davis Technologies, Alachua, FL, USA) was used to generate specific acoustic stimuli and to amplify, measure, and display the evoked brainstem responses of anesthetized mice $(10 \%$ chloral hydrate, concentration $=0.0045 \mathrm{ml} / \mathrm{g}$ body weight). Animals were kept warm with a heating pad in a soundproof shielded room during ABR recordings. Subdermal needle electrodes were inserted at the vertex and ventrolaterally to both ears of anesthetized mice. Specific auditory stimuli (broadband click and pure-tone pips of $1,2,4,8$, and $16 \mathrm{kHz}$ ), with 20 beats/second recurrence rate, 1024 average superposition times, $20 \mathrm{~min}$ of scanning time, and $100-3000 \mathrm{~Hz}$ of filtering wave bandwidth, were delivered binaurally through plastic tubes in the ear canals. Evoked brainstem responses were amplified and averaged and their wave patterns electronically displayed. Auditory thresholds were obtained for each stimulus by varying the sound pressure level (SPL) at 5-dB steps up and down in order to identify the lowest level at which an ABR pattern could be recognized. ABR thresholds were determined for each stimulus frequency by identifying the lowest intensity producing a reproducible $\mathrm{ABR}$ pattern on the computer screen (at least two consistent peaks).

\section{Preparations of cochlear hair cells}

The mice were sacrificed by cervical dislocation and further decapitation. Next, the organ of Corti was dissected and directly placed in an imaging chamber filled with Hank's Balanced Salt Solution without calcium (HBSS without $\mathrm{Ca}^{2+}$ ) containing (in $\mathrm{mM}$ ): [5.36 KCl, $141.7 \mathrm{NaCl}, 1 \mathrm{MgCl}_{2}, 0.5 \mathrm{MgSO}_{4}, 10$ HEPES, 3.4 L-Glutamine, and 6.9 D-Glucose, $\mathrm{pH} 7.4$ ] at $37^{\circ} \mathrm{C}$.

\section{FM1-43 loading and imaging}

FM1-43 (Invitrogen, U.S.A) was dissolved in DMSO to obtain a stock solution with a concentration of $1 \mathrm{mM}$. Time-lapse z-stack series along the Hair cells (HCs) longitudinal axis were collected using Delta Vision Microscopy equipped with a water immersion objective $(63 \times, 0.9)$ (Delta Vision Microscopy Imaging Systems, UK). All solutions were carbogen-charged and prewarmed at $37{ }^{\circ} \mathrm{C}$ before the experiments. For labeling at room temperature, the HCs was pre-incubated for $2 \mathrm{~min}$ in a plastic plate containing HBSS without $\mathrm{Ca}^{2+}$, followed by incubation in HBSS removal of $\mathrm{Ca}^{2+}, 1.3 \mathrm{mM}$ FM 1-43 for $5 \mathrm{~min}$ at room temperature. In this study, both of the IHCs and OHCs were labeled by fluorescence FM 1-43 dye in the observations of fluorescence microscopy. The IHCs and OHCs were dissected from normal mice at ages between P29-30, and incubated for 1000 seconds at room temperature in a bathing solution containing $1 \mathrm{mM}$ FM 1-43. The sites and quantity of the dye entry into hair cells were observed using fluorescence microscopy, respectively. All images were obtained with identical settings on a confocal DeltaVision Microscopy Imaging 


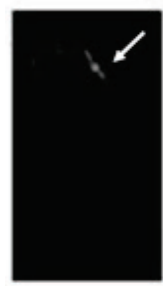

50

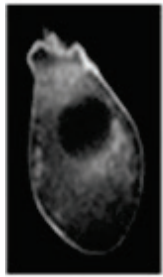

550

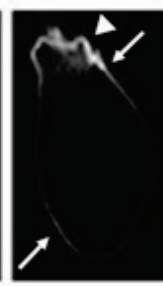

100

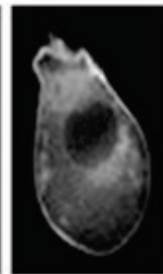

600

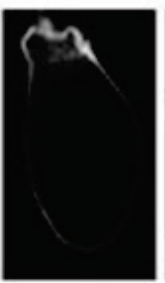

150

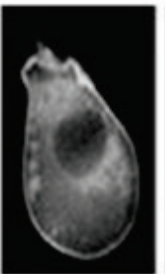

650

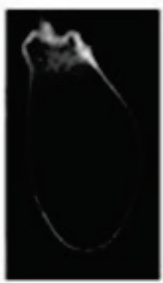

200

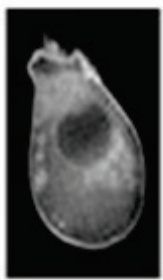

700

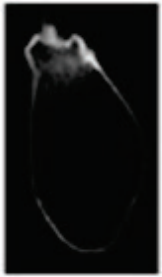

250

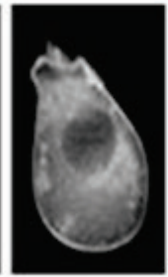

750

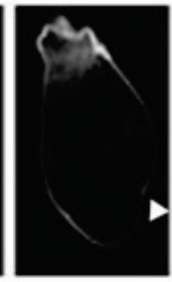

300

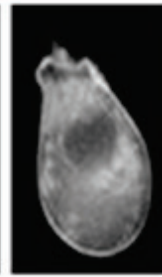

800

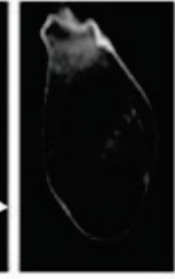

350

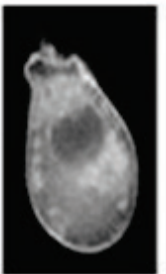

850
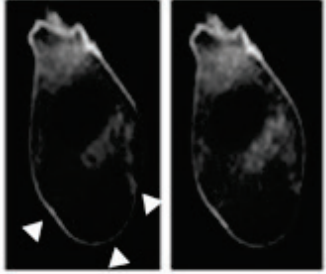

450

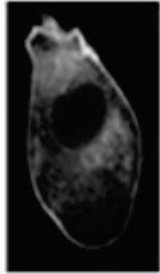

400
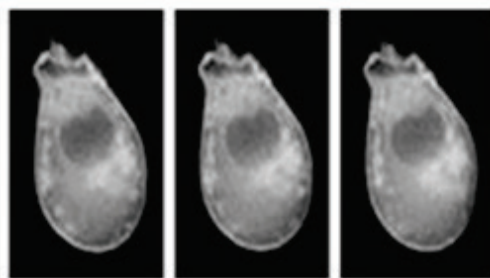

900

\section{0}

1000

Fig. 1. FM1-43 dye uptake in the IHCs in adult mice. At $50 \mathrm{~s}$ after dye was loaded, bright fluorescence was initially seen in the apical area of IHCs observed by live confocal imaging (indicated by arrow). At 100s, stained apical surfaces enlarged from the original fluorescence spot (indicated by arrowhead and arrow). Moreover, additional stained membrane area was found at the basolateral region at the same time point (indicated by arrow, left lower side, 100s). At the 300s, the third independent spot fluorescence stained was identified at the basolateral area (arrowhead). At the 350s, two fluorescence stained area appeared inside the cells, one of the areas was in the adjoining region of the apical membrane, and another area was observed to be close to the basolateral membrane with stained spot. At the $400 \mathrm{~s}$, the third fluorescence stained area in the cell was seen to connect other basolateral fluorescence spot (arrowheads). From 450s to 1000s, all stained fluorescence of the cell strengthened significantly.

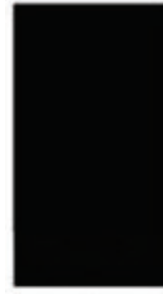

50

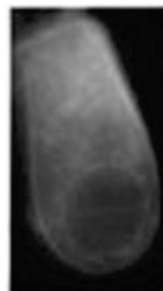

550

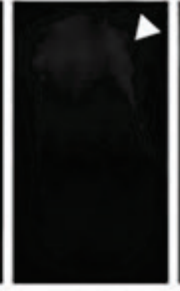

100

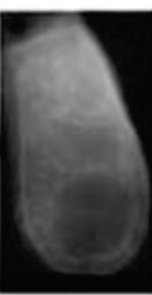

600

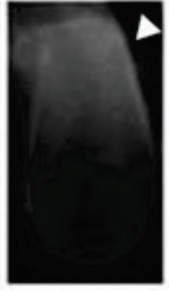

150

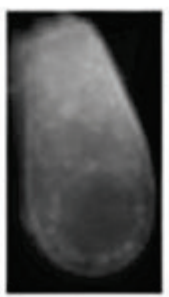

650

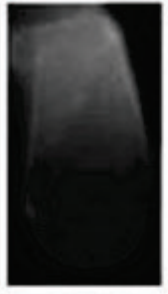

200

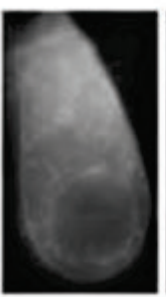

700

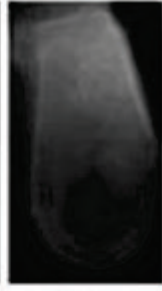

250

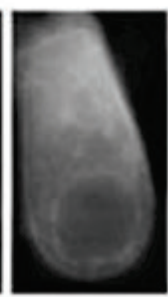

750

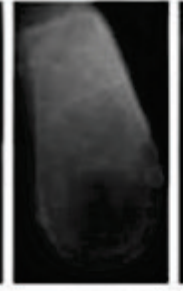

300

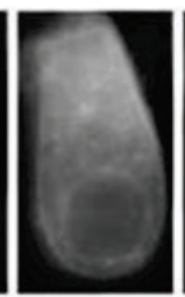

800

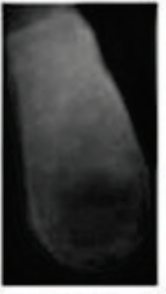

350

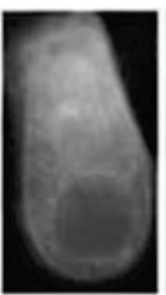

850

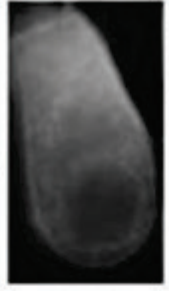

400

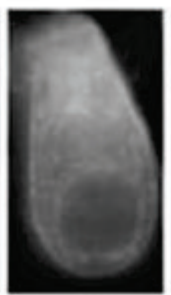

900

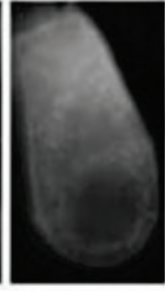

450

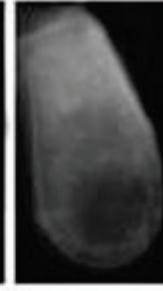

500
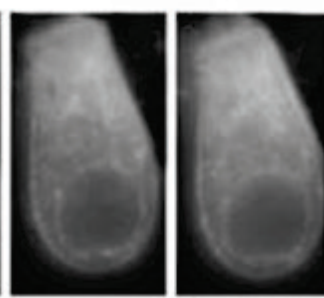

950

1000

Fig. 2. FM1-43 dye uptake in adult $\mathrm{OHC}$ in mice. Fluorescence, which is an indicative of endocytosis in OHCs, was initially observed at $100 \mathrm{~s}$ after dye loading (indicated by arrowhead, 100s). Bright fluorescence area was identified in the apical membrane (shown by arrowhead, 150s). From 200s to 1000s, fluorescence stained membrane surface in OHCs enlarged and diffused significantly towards insides of the cell, fluorescence intensities were identified uniformly.

Systems (UK). FM dyes were excited with the $488 \mathrm{~nm}$ line of a laser and their emission was detected in the range of 500-700 $\mathrm{nm}$ by a photomultiplier tube (PMT).

\section{Statistical analysis}

All data are presented as means \pm SEM. Data were examined using a one-way ANOVA with StudentNewman-Keuls test to determine the differences between groups. All statistical analyses were performed using the SPSS16.0 statistical software package. A $p$ value $<0.05$ was considered statistically significant. 


\section{Results}

\section{The route of FM 1-43 dye entry into the IHCs}

Fluorescence microscopy showed that after its loading, FM1-43 dye initially appeared at the apex of IHC (Fig. 1, 50s, indicated by arrow). However, the site with highest fluorescence intensity is not the same area where apical stereocilia are located (Fig. 1, 100s and150s, indicated by arrowhead). Up to $50 \mathrm{~s}$ after the initial loading of FM1-43 dye, the bright spot (characterized by bright fluorescence) was observed only at the right side in the apical membrane of IHC. This indicates it to be the most likely location for the entry of the dye into the cells, further suggesting this location to be the initial and unique area where the endocytosis occurred (Fig. 1, 50s, arrow). Next, we found that all the apex area and stereocilia were labeled with bright fluorescence at $100 \mathrm{~s}$ after the initial loading of the dye (Fig. 1, 100s, arrowhead and arrow), suggesting that the apical area labeling may expand significantly from the original spot of entry. In this study, we have identified independent additional area near the basolateral region, where FM1-43 dye entered into IHC (Fig. 1, $100 \mathrm{~s}$, indicated by arrow). The difference in the intensity of fluorescence between the apical and the basolateral spot is relatively small, indicating that endocytosis also occurs in the basolateral membrane of IHC.

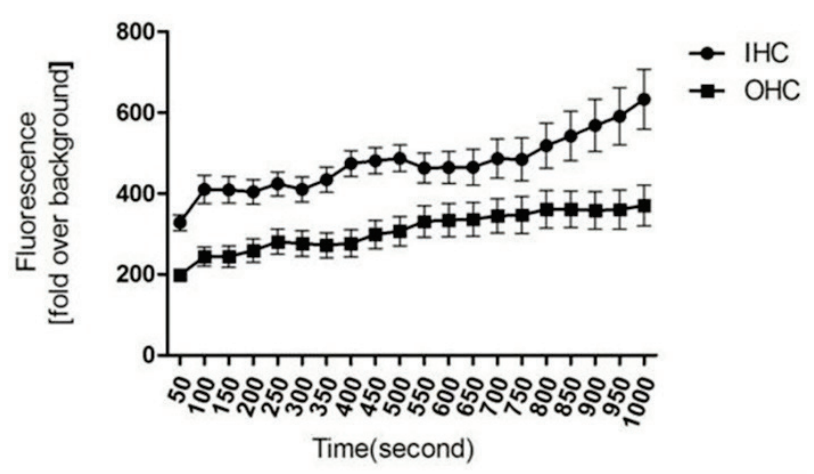

Fig. 3. Quantitative analysis of capacities of FM1-43 dye uptake in the IHCs and OHCs of adult mice. The fluorescence intensities for FM1-43 dye uptake into IHCs were significantly higher than those observed for OHCs fluorescence intensity of FM1-43 dye in IHCs reveals more positive (linear) correlation between the quantum of dye uptake and the duration of exposure time, as compared to that observed in OHCs. Data was collected from 5 IHCs and $\mathrm{OHCs}$, respectively.

The third independent spot with bright fluorescence was observed at 300s after the initial dye loading, this spot was located in the basolateral membrane below the nuclei of IHCs. Due to that the fluorescence intensity at the adjacent membranes were significant weaker (Fig. 1, 300s, arrowhead). Our study showed that there could be three independent locations in IHC responsible for FM1-43 dye entry into the cell, suggesting that activity of endocytosis independently exist in the three different regions of IHC membrane.

The dye was observed to diffuse rapidly towards other parts of membrane and cytoplasm after its internalization into the cells. Our study showed that most parts of membrane and membrane of granules in cytoplasma were labeled with bright fluorescence (Fig. 1, 300s-1000s), suggesting that velocities of dye diffusing at different parts of IHCs are quite distinct.

\section{The pattern of FM 1-43 dye entry into the OHCs}

In this study, we firstly estimated the velocity of FM 1-43 dye entry into OHCs. We observed that FM1-43 dye entry into OHCs quite differently compared with that of IHC in the same preparations. Apparent areas with positive dye staining were initially found at $150 \mathrm{~s}$ after the initial dye loading (Fig. 2, $150 \mathrm{~s}$, as indicated by the arrowhead), suggesting a significant lower velocity of FM1-43 uptake compared with that of IHC. Second, we examined the sites of entry of FM 1-43 into OHCs. Our study showed that one site of apical area is likely the only location on the surface area of OHC in the onset of dye entry into cell (Fig. 2, 150s, arrowhead). After that, it is likely most areas of $\mathrm{OHC}$ surface were uniformly stained via FM 1-43 dye. From $150 \mathrm{~s}$ to $1000 \mathrm{~s}$, several aggregated bright fluorescent stained spots appeared gradually inside the cell, this might suggest the presence of massive aggregated granules or/and endosomes, which were stained with the dye (Fig. 2).

Quantitative analysis of capacity comparable between IHC and OHC in the uptake of FM1-43 dye

Considering the distinct roles of OHCs and IHCs in the hearing pathway, there might be different properties between two types of hair cells in the uptake of dye. We then examined the quantum and velocity of dye uptake in the two types of cells. During of the total exposure time (up to $1000 \mathrm{~s}$ ), dye uptake into the IHCs and OHCs were dynamically captured and quantified in the interval of $50 \mathrm{~s}$, the fluorescence intensities in both of IHC and $\mathrm{OHC}$ were normalized for comparison. And we found that initial fluorescence intensity of dye uptake in IHC was more than $50 \%$ higher than that of $\mathrm{OHC}$ (Fig. 3, 50s, $\mathrm{P}<0.05$ ). Further, we quantified the 
fluorescence intensities at all the time points in the entire duration for both IHC and OHC. At all the time points, fluorescence intensities of FM1-43 dye in IHCs were significantly stronger than those observed for $\mathrm{OHCs}$ (Fig. 3, $100 \mathrm{~s}-1000 \mathrm{~s}, \mathrm{P}<0.05$ ). At the time corresponding to $1000 \mathrm{~s}$, after initial loading, the intensity of fluorescence of FM1-43 dye in IHCs was observed to be over 600 , whereas, the intensity of fluorescence dye in $\mathrm{OHC}$ is less than 300 (Fig. 3, $1000 \mathrm{~s}, \mathrm{P}<0.01$ ). Our results suggest that the capacity of FM1-43 dye uptake in IHCs is remarkable higher than that of OHCs.
A

(2)

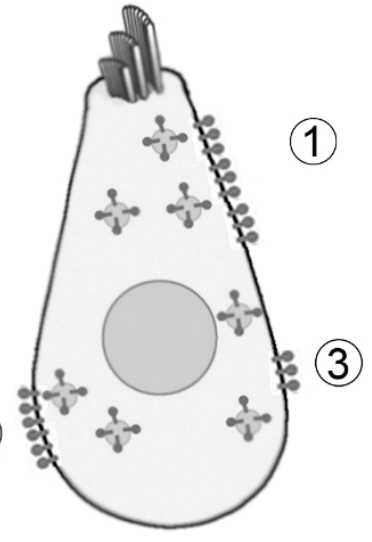

\section{Discussion}

Our studies demonstrated that FM1-43 dye uptake in IHCs and OHCs present different patterns, such as distinct sites of dye uptake, and different velocities of dye entry into the cells. In general, IHC was shown to have a stronger capacity of dye uptake in comparison with OHC. The different patterns of FM1-43 dye uptake in IHC and $\mathrm{OHC}$ may reflect distinct roles of two types of cochlear hair cells, contributing largely to development and maintenance of normal hearing.

Previous studies have demonstrated the use of FM1-43 dye in real time tracing of the structures undergoing endocytosis and exocytosis in in living cell (Brumback et al. 2004, Iwabuchi et al. 2014). Three reasons were proposed to allow FM dyes can be used for the estimations of cellular activities, such as endocytosis, exocytosis, as well as vesicle trafficking are as follows: i) FM dye molecules are permanently positively charged $\left(2^{+}\right)$, resulting in that FM dyes only selectively stain external leaflet of the lipid bilayer of cell membrane. Therefore, FM dyes are unable to diffuse through membranes of healthy neuron. ii) The dye "destains" when the cells are washed with dye-free buffer, Therefore, the presence of fluorescence dye inside the cell is usually resultant of ingestion of endosomes that were stained by FM dye. In this study, we observed fluorescence within both IHC and $\mathrm{OHC}$, there is most likely due to the internalization of the stained
Fig. 4. Schematic representation of different patterns of endocytosis between IHC and OHC. (A) In IHC, bright fluorescence of FM1-43 dye was initially seen at apical area (1), followed by relatively attenuated fluorescence appearing at the basolateral region (2). Next, a weaker fluorescencewas observed at the contralateral basolateral area (3). (B) In $\mathrm{OHC}$, the fluorescence was also initially seen at the apical membrane (1). Subsequently the most areas of $\mathrm{OHC}$ surface were stained uniformly FM 1-43 dye (2). Furthermore, quantitative analyses suggest that the amount of FM1-43 dye uptake in IHC is remarkably higher than that observed for $\mathrm{OHC}$, during the same experimental time limit. endosomes/and or granules. iii) FM dyes cannot be labeled via fluorescent in water, however, if the dye partition into a hydrophobic environment, the total quantity of labeling fluorescent intensity enhances more than hundreds times.

Moreover, this property even can be used to estimate exocytosis. When secretory vesicles fuse with the surface membrane of the cell undergoing exocytosis, the FM dye can stain the newly bulged membrane via diffusing through the fusion pore, leading to a significant enhancement of fluorescence intensity (Brumback et al. 2004). This result has been confirmed using the whole cell capacitance recordings in the cells undergoing exocytosis. When the area of membrane being added to the cell surface via exocytosis is greater than that of membrane being retrieved by endocytosis, the cell capacitance enhances. Conversely, when the amount of endocytosis is greater than exocytosis, the cell capacitance reduce (Rituper et al. 2013). When a cell that is undergoing exocytosis is stained with FM1-43, the plasma membrane fluorescence increases due to vesicles fusion with the surface of the cell. However, when vesicles subsequently undergo endocytosis, the cell fluorescence remains unchanged as the same amount of dye remains present in the vesicles despite their fusion with surface membrane or internalization via endocytosis.

In IHC, net apical FM1-43 uptake exceeds basolateral uptake, suggesting a higher capacity of endocytosis in apical area compared to that in basolateral 
area (Griesinger et al. 2002). Our data is consistent with data that we found a quite fast and stronger fluorescent intensity in apex of IHCs. In addition, our study revealed a weak, but distinct, FM1-43 dye uptake across the basolateral membrane of IHCs in spite the fact that FM1-43 uptake in apical region gave larger fluorescent intensities in the same cell. It is well known that most activities of synaptic releasing occur in the basolateral membrane of IHC, raising a question whether the endocytosis in the basolateral membrane can meet huge demands of synaptic activities in IHC? Considering a moderate level of FM1-43 uptake across the basolateral membrane of IHC, it is reasonable to assume that there could be a cross talk between the base and apex of the IHC that can replenish basolateral membrane retrieval to provide sufficient synaptic vesicles near/or at the synaptic releasing sites. Parsons et al. demonstrated this assumption to be true using capacitance measurements in IHCs (Parsons et al. 1994). IHCs were found to have an abundant network of microtubules stretching from the apical surface to basolateral area (Steyger et al. 1989, Furness et al. 1990). Further, a large number of vesicles in the apex were found contacted to microtubules in hair cells (Kachar et al. 1997). indicating a possibility that vesicular transport from apical area to basolateral location, which is responsible spatially for the activities of synaptic releasing. Interestingly, our study also showed additional and independent fluorescence stained spots at the base of IHC, suggesting the occurrence of synaptic releasing mainly at the basal area of the IHC.

Comparing with that whole IHC was fully fluorescence stained within min, however, OHC presented a slow and weak pattern of FM1-43 dye uptake. These differences observed between IHC and OHC are consistent with previous reports of cochlear IHCs are being the true sensory neurons, with a requirement of maintaining exceedingly high firing rates (Palmer and Russell 1986). The rate of IHC afferent activities required tremendous demands of vesicles replenishment (Moser and Beutner 2000). However, because it is not necessary to have high firing rates in $\mathrm{OHC}$ afferents, large amount of apical preservations of transmitters might not be needed (Kaneko et al. 2006).

It should be noted that it remains unclear whether mechanotransducer channels are the major entries for FM1-43 dye uptake in cochlear hair cells. Several studies proposed that the cytoplasm of cochlear hair cells becomes labeled via FM1-43 very fast by entering through mechanotransducer channels, which are usually found in apex and sterecilla (Nishikawa and Sasaki, 1996, Gale et al. 2001). However, another group showed an opposite evidence concluding that FM dye uptake in cochlear hair cells does not necessarily involve a passage through the mechanotransducer channels (Griesinger et al. 2002). Thus, further studies are needed to approach the truth of entry sites for FM dye in cochlear hair cells.

\section{Conflict of Interest}

There is no conflict of interest.

\section{Acknowledgements}

This work was supported by Beijing Natural Science Foundation [grant number 5122040]. The funder had no role in study design, nor in the collection, analysis and interpretation of data, nor in the writing of the report, neither in the decision to submit the article for publication.

\section{References}

BRUMBACK AC, LIEBER JL, ANGLESON JK, BETZ WJ: Using FM1-43 to study neuropeptide granule dynamics and exocytosis. Methods (San Diego, Calif.) 33: 287-294, 2004.

COUSIN MA: Integration of synaptic vesicle cargo retrieval with endocytosis at central nerve terminals. Front Cell Neurosci 11: 234, 2017.

FURNESS DN, HACKNEY CM, STEYGER PS: Organization of microtubules in cochlear hair cells. $J$ Electron Microsc Tech 15: 261-279, 1990.

GALE JE, MARCOTTI W, KENNEDY HJ, KROS CJ, RICHARDSON GP: FM1-43 dye behaves as a permeant blocker of the hair-cell mechanotransducer channel. J Neurosci 21: 7013-7025, 2001.

GRIESINGER CB, RICHARDS, CD, ASHMORE JF: Fm1-43 reveals membrane recycling in adult inner hair cells of the mammalian cochlea. $J$ Neurosci 22: 3939-3952, 2002.

GRIESINGER CB, RICHARDS CD, ASHMORE JF: Apical endocytosis in outer hair cells of the mammalian cochlea. Eur J Neurosci 20: 41-50, 2004. 
IWABUCHI S, KAKAZU Y, KOH JY, GOODMAN KM, HARATA NC: Examination of synaptic vesicle recycling using FM dyes during evoked, spontaneous, and miniature synaptic activities. J Vis Exp 85: 50557-50367, 2014.

KACHAR B, BATTAGLIA A, FEX J: Compartmentalized vesicular traffic around the hair cell cuticular plate. Hear Res 107: 102-112, 1997.

KAMIN D, REVELO NH, RIZZOLI SO: FM dye photo-oxidation as a tool for monitoring membrane recycling in inner hair cells. PloS One 9: 88353-88365, 2014.

KANEKO T, HARASZTOSI, C, MACK AF, GUMMER AW: Membrane traffic in outer hair cells of the adult mammalian cochlea. Eur J Neurosci 23: 2712-2722, 2006.

MERCHAN-PEREZ A, LIBERMAN MC: Ultrastructural differences among afferent synapses on cochlear hair cells: correlations with spontaneous discharge rate. J Comp Neurol 371: 208-221, 1996.

MEYER J, MACK AF, GUMMER AW: Pronounced infracuticular endocytosis in mammalian outer hair cells. Hear Res 161: 10-22, 2001.

MOSER T, BEUTNER D: Kinetics of exocytosis and endocytosis at the cochlear inner hair cell afferent synapse of the mouse. Proc Natl Acad Sci U S A 97: 883-888, 2000.

NEEF J, JUNG S, WONG AB, REUTER K, PANGRSIC T, CHAKRABARTI R, KUGLER S, LENZ C, NOUVIAN R, BOUMIL RM, FRANKEL WN, WICHMANN C, MOSER T: Modes and regulation of endocytic membrane retrieval in mouse auditory hair cells. J Neurosci 34: 705-716, 2014.

NISHIKAWA S, SASAKI F: Internalization of styryl dye FM1-43 in the hair cells of lateral line organs in Xenopus larvae. J Histochem Cytochem 44: 733-741, 1996.

PALMER AR, RUSSELL IJ: Phase-locking in the cochlear nerve of the guinea-pig and its relation to the receptor potential of inner hair-cells. Hear Res 24: 1-15, 1986.

PARSONS TD, LENZI D, ALMERS W, ROBERTS WM: Calcium-triggered exocytosis and endocytosis in an isolated presynaptic cell: capacitance measurements in saccular hair cells. Neuron 13: 875-883, 1994.

RITUPER B, GUCEK A, JORGACEVSKI J, FLASKER A, KREFT M, ZOREC R: High-resolution membrane capacitance measurements for the study of exocytosis and endocytosis. Nat Protoc 8: 1169-1183, 2013.

STEYGER PS, FURNESS DN, HACKNEY CM, RICHARDSON GP: Tubulin and microtubules in cochlear hair cells: comparative immunocytochemistry and ultrastructure. Hear Res 42: 1-16, 1989.

XIE Z, LONG J, LIU J, CHAI Z, KANG X, WANG C: Molecular mechanisms for the coupling of endocytosis to exocytosis in neurons. Front Mol Neurosci 10: 3389-3397, 2017. 